\title{
Expression, regulation and function of miR-495 in healthy and tumor tissues (Review)
}

\author{
HONGLI CHEN $^{1}$, XIAMAN WANG ${ }^{1}, \mathrm{JU} \mathrm{BAI}^{1}$ and AILI HE $\mathrm{HE}^{1,2}$ \\ ${ }^{1}$ Department of Hematology, Second Affiliated Hospital of Xi'an Jiaotong University; \\ ${ }^{2}$ National-Local Joint Engineering Research Center of Biodiagnostics and Biotherapy, \\ Xi'an Jiaotong University, Xi'an, Shaanxi 710004, P.R. China
}

Received October 2, 2015; Accepted November 4, 2016

DOI: $10.3892 / \mathrm{ol} .2017 .5727$

\begin{abstract}
MicroRNA-495 (miR-495) is a small non-coding RNA encoded by a gene located on chromosome 14 (14q32.31). Its expression is regulated by the transcription factors EF12 and EF47, in addition to promoter methylation status and the fusion oncoprotein mixed-lineage leukemia-AF9. Previous studies suggest that miR-495 is involved in various developmental, immunological and inflammatory processes in healthy tissue, and in the proliferation, invasion, metastasis and drug resistance of cancer cells. The role miR-495 serves in tumors is controversial. miR-495 primarily functions as a tumor suppressor; however, in a number of cases it acts as an oncogene. miR-495 has potential applications as a diagnostic and prognostic marker, and as a therapeutic target for genetic and pharmacological manipulation in the treatment of various diseases.
\end{abstract}

\section{Contents}

1. Introduction

2. Genetic structure and regulation of miR-495

3. Role of miR-495 in healthy tissue

4. Role of miR-495 in tumors

5. Conclusions

\section{Introduction}

MicroRNAs (miRNAs or miRs) are small, non-coding single-stranded RNAs that contain 22 nucleotides. They negatively regulate gene expression at the post-transcriptional level by inducing translational inhibition or mRNA degradation (1).

Correspondence to: Professor Aili He, Department of Hematology, Second Affiliated Hospital of Xi'an Jiaotong University, 157 West Five Road, Xi'an, Shaanxi 710004, P.R. China

E-mail: heaili@mail.xjtu.edu.cn

Key words: miRNA-495, 14q32.31 cluster, expression regulation, function
miRNAs are encoded by their own genes or the introns of host genes (2) and mature miRNAs are generated through a multistage process. Initially, the miRNA gene is transcribed by RNA polymerase II or III in the nucleus to form primary miRNA (pri-miRNA) (3). pri-miRNA is then processed into a characteristic stem-loop sequence called precursor miRNA (pre-miRNA) by the ribonuclease III enzyme Drosha (4). The pre-miRNA is subsequently transported into the cytoplasm by exportin-5 and cleaved to form a short-lived double-stranded RNA. One strand is typically degraded and the mature miRNA strand is incorporated into an Argonaute protein-containing complex called the RNA-induced silencing complex (5).

miRNAs serve essential roles in physiological and pathological processes, by incomplete or complete complementary binding to the $3^{\prime}$ untranslated region of their target mRNAs. In certain circumstances, miRNA genes are closely located and form an miRNA cluster (6). A large number of clustered miRNA genes have been identified, in which the expression and regulatory function of the clustered miRNAs are cooperative and share cis-regulatory elements. With increasing numbers of miRNA being identified, the size of this miRNA cluster will increase; however, the regulatory mechanisms underlying the expression of these miRNAs remains unclear. The human $14 q 32.31$ gene cluster is located in an imprinted region of the genome and contains a number of genes encoding miRNAs, the majority of which exhibit similar biological activities (7). The miRNA miR-495, is a member of the 14q32.31 miRNA cluster and has been well investigated. Along with other miRNAs in the $14 q 32.31$ cluster, miR-495 is involved in promoting the development of normal tissue and regulating proliferation, apoptosis, metastasis and chemosensitivity in cancer cells. Therefore, in the present review the regulation of expression of miR-495, its roles in development, immunity and inflammation in normal tissue, and its regulatory function in cancer were investigated.

\section{Genetic structure and regulation of miR-495}

The miR-495 gene is located on 14q32.31 and contains a single exon. The miRNA gene cluster 14q32.31 contains the gene for miR-495, along with the genes encoding miR-299-5p, miR-376a, miR-376c, miR-300, miR-380, miR-494, miR-1197 and others (Fig. 1). miRNAs in this cluster perform similar activities in normal and diseased tissues $(8,9)$. The miR-495 
pair, miR-495-3p and miR-495-5p are cleaved from the 3'- and 5'-strands of the stem-loop structure of pre-miRNA-495, respectively $(10,11)$. Ectopic miR-495 serves an essential role in tumor tissues. Previous studies have demonstrated that miR-495 expression is associated with promoter methylation status and transcriptional factors, such as EF12 $(12,13)$.

In gastric cancer (GC), promoter hypermethylation has been demonstrated to induce deregulation of miR-495 (12). Furthermore, low-level cytosine-phosphate-guanine methylation of the miR-495 gene and expression of miR-495 were recovered following treatment with 5-Aaza-2' deoxycytidine, a DNA methyltransferase inhibitor (12). In addition, transcriptional factors serve a role in regulating the expression of miR-495. In breast cancer stem cells, increased expression of miR-495 was demonstrated to be induced by the transcriptional factors E12 and E47 (13). Expression of E12/E47 is associated with miR-495 promoter activity (13). The overexpression of the fusion protein mixed-lineage leukemia (MLL)-AF9 was demonstrated to significantly downregulate expression of miR-495 (14). Thus, the MLL-AF9 fusion protein potentially acts as a silencer of miR-495. Further studies are warranted to explore other mechanisms regulating the expression of miR-495.

\section{Role of miR-495 in healthy tissue}

Role of miR-495 in development. miR-495 is involved in the development of stem cells, cartilage and pancreatic acinar cells. Through specifically targeting DNA methyltransferase $3 \mathrm{a}$, miR-495 promotes the mesendoderm differentiation of mouse embryonic stem cells during the first two days of embryoid body development (15). miR-495 is also a significant regulator of chondrogenic differentiation in human mesenchymal stem cells. Potentially, miR-495 induces a higher expression of SRY-box 9 (SOX9), which ultimately contributes to chondrogenic differentiation (16). SOX9 is an important regulator of mesenchymal condensation in the early stages of cartilage differentiation (17). Hence, the function of SOX9 in chondrogenic development may be miR-495 dependent.

miR-495 is an essential factor for normal pancreatic development. Along with the miRNA let-7b, miR-495 induces the differentiation and prevents metaplasia of pancreatic acinar cells by repressing hepatocyte nuclear factor 6 (18). In humans, miR-495 and other miRNAs in the 14q32.31 cluster also take part in the differentiation of hematopoietic stem cells and neovascularization $(19,20)$.

Role of miR-495 in inflammation and immunity. In regards to inflammatory diseases, miR-495 is primarily associated with Crohn's disease (CD). Mutations in the coding region of nucleotide binding oligomerization domain containing protein 2 (NOD2) are strongly associated with CD pathogenesis. The expression of proinflammatory [interleukin (IL)-6, IL-8 and TNF- $\alpha$ ] and anti-inflammatory (IL-10) factors are influenced by NOD2 (21). In the human colonic epithelial cell line HCT116, NOD2 is the downstream target of miR-495 (22). Subsequently, upregulated NOD2 causes inflammation by inducing the expression of IL-8 and C-X-C motif chemokine ligand $3(11,23)$. This partially explains the mechanisms underlying inflammation in CD. Furthermore, the activation of platelets serves a significant role in the development of inflammatory bowel disease (24). miR-495 activates platelets in CD by upregulating kelch like family member 5 , the activated platelets then produce a variety of inflammatory factors and cause local vascular inflammation (25). This demonstrates a further inflammatory mechanism in CD.

miR-495 serves a role in immunity. NOD2 effects innate immunity through its expression in antigen-presenting cells. (26). As ectopic miR-495 affects the expression of NOD2, this indicates that miR-495 serves a role in the immune response. A role of miRNAs in adaptive immunity was identified by Choi et al (10), through infecting mice with the highly virulent avian H5N2 virus. Nine miRNAs that were upregulated in the lungs following this were identified, including miR-495. Four other highly expressed miRNAs were selected and identified to be associated with the immune response, apoptosis and viral replication. However, the activity of miR-495 was not studied and further studies are required to confirm that miR-495 serves a role in the immune response.

\section{Role of miR-495 in tumors}

Tumorigenesis is a complex process, which progresses at a cellular, genetic and epigenetic level, and ultimately leads to uncontrolled cell growth and abnormal apoptosis (27). Previous studies have demonstrated that miR-495 serves essential roles in promoting proliferation, invasion, metastasis and chemosensitivity of solid tumors and hematological tumor cells.

Ectopic miR-495 regulates cell proliferation, invasion, metastasis and tumorigenesis in solid tumors. miR-495 serves an anti-tumor role in the majority of solid tumors. Phosphatase of regenerating liver-3 (PRL-3) has been associated with the invasion and metastasis of gastric cancer cells (28). In GC tissues, miR-495 expression is associated with PRL-3 upregulation. Experiments in gastric cancer cell lines demonstrated that PRL-3 is a downstream target of miR-495, and that the invasion and metastasis of tumor cells was inhibited by transfection of miR-495 mimics $(12,29)$.

miR-495 acts as a tumor suppressor and is downregulated in non-small cell lung cancer (NSCLC) (30). Increased metastasis associated 1 family member 3 (MTA3) is a recognized risk factor for lymph node metastasis in NSCLC. Deregulation of miR-495 could influence the metastasis of NSCLC by targeting MTA3 mRNA for degradation. Additionally, in glioblastoma multiforme U-251 cells loss of miR-495 increases glucose uptake and lactate secretion, which promotes proliferation, as miR-495 is no longer targeting cyclin-dependent kinase 6 for degradation (31). Finally, downregulation of miR-495 has been associated with tumorigenesis in metastatic prostate cancer (9). These observations support the hypothesis that miR-495 acts as a tumor suppressor.

Increased expression of miR-495 has been associated with a carcinogenic effect in a number of solid malignancies. In breast cancer stem cells, epithelial-cadherin (E-cadherin) and DNA damage inducible transcript 4 (REDD1) have been investigated, with results suggesting they are direct targets of miR-495 (13). Overexpression of miR-495 leads to the downregulation of E-cadherin and the subsequent promotion of cell invasion (13). Furthermore, decreased REDD1 


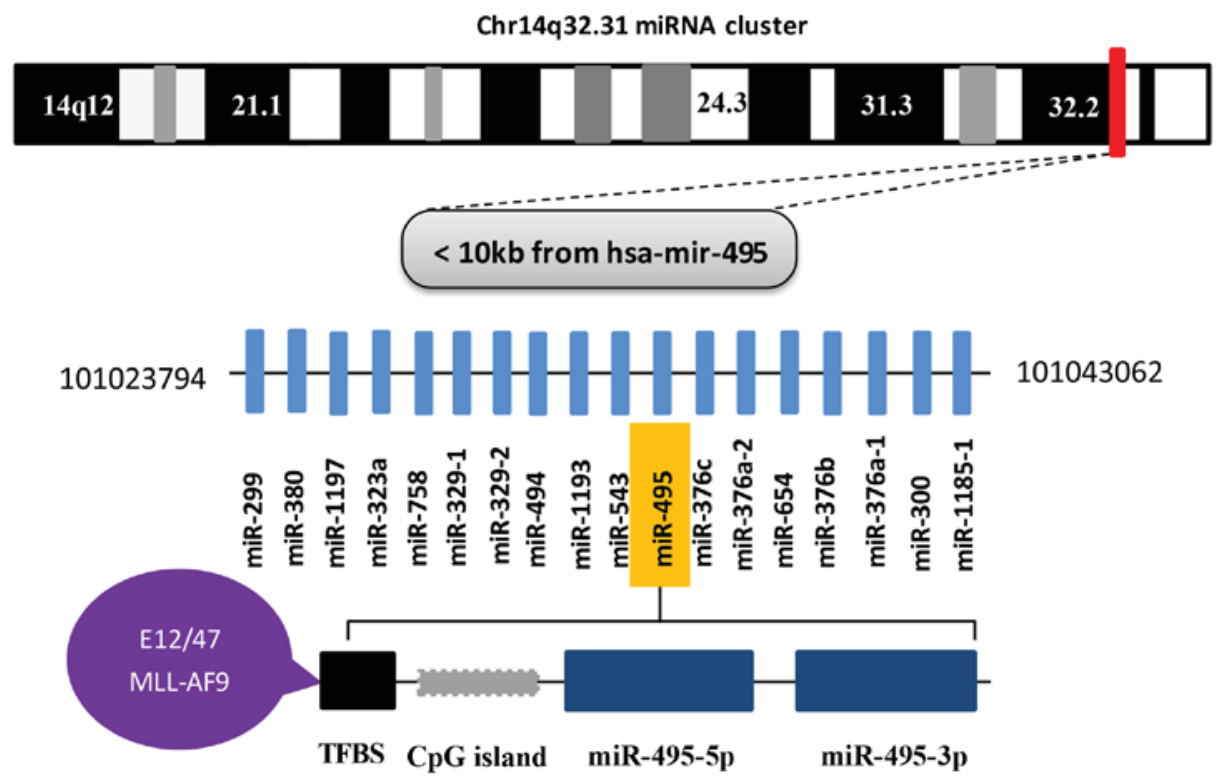

Figure 1. Location of the 14q32.31 miRNA cluster on chromosome 14 ( $<10 \mathrm{~kb}$ from hsa-miR-495), the miRNA genes it contains and the structure of the gene encoding miR-495. miR-495 has two mature types, miR-495-5p and miR-495-3p. The high-level CpG methylation of the miR-495 gene in the promoter region results in the deregulation of miR-495. E12 and E47 induce, and MLL-AF9 represses, the transcription of miR-495 through binding to the TFBS. CpG, cytosine-phosphate-guanine; MLL, mixed-lineage leukemia; TFBS, transcription factor binding site.

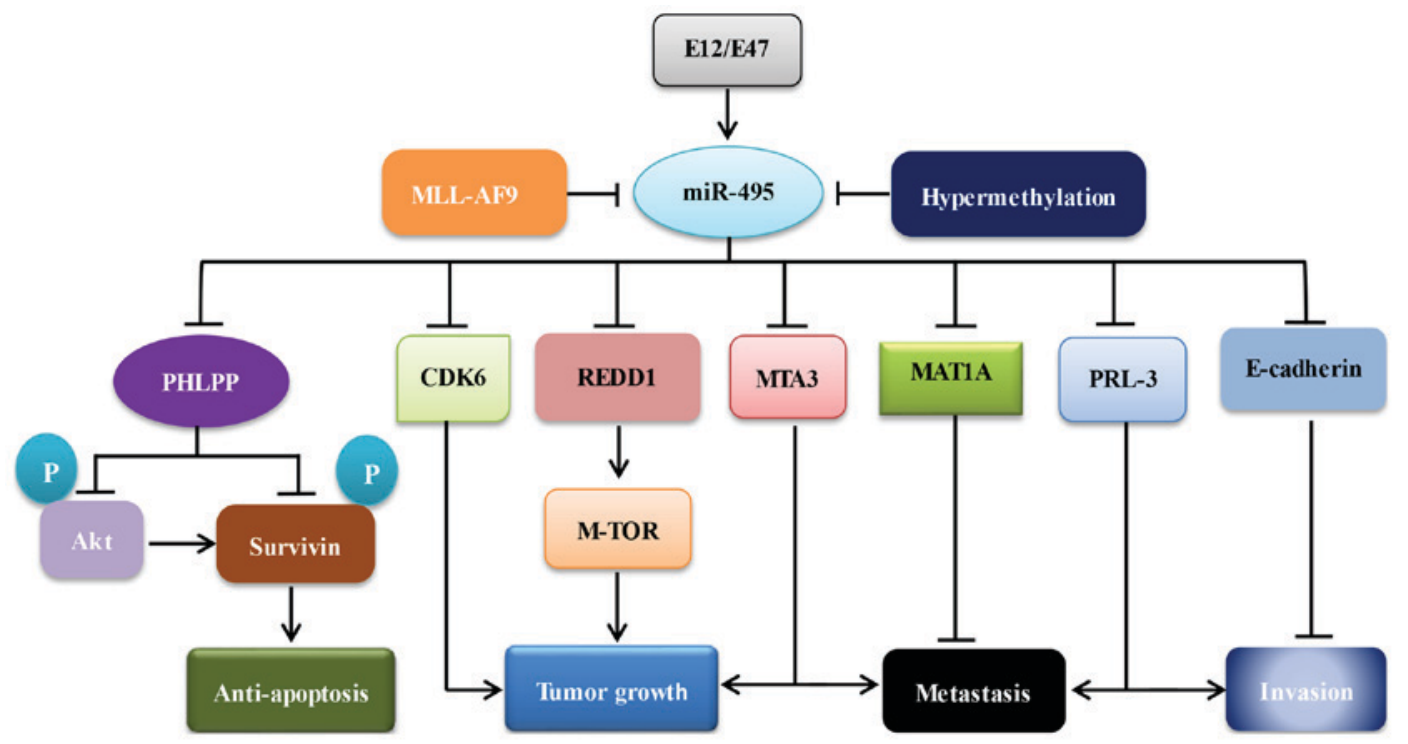

Figure 2. miR-495 is regulated by the transcription factors EF12 and EF47, promoter methylation status and the fusion oncoprotein MLL-AF9. miR-495 negatively modulates the expression of its target mRNAs (PHLLP, CDK6, REDD1, MTA3, PRL-3, E-cadherin and MAT1A) and is involved in anti-apoptosis, tumor growth, metastasis and invasion through the AKT, mTOR and E-cadherin signaling pathways. MLL, mixed-lineage leukemia; PHLLP, PH domain and leucine rich repeat protein phosphatase; CDK6, cyclin dependent kinase 6; REDD1, DNA damage inducible transcript 4; MTA3, metastasis associated 1 family member 3; PRL-3, phosphatase of regenerating liver-3; E-cadherin, epithelial-cadherin; MAT1A, methionine adenosyltransferase 1A; P, phosphorylation; AKT, AKT serine/threonine kinase; mTOR, mechanistic target of rapamycin.

expression, due to overexpression of miR-495, results in the release of inhibition of the mechanistic target of rapamycin signaling pathway, promoting the proliferation of cancer cells in hypoxic conditions (13). Additionally, miR-495 serves a role in the growth, apoptosis and metastasis of hepatocellular carcinoma (HCC) cells (32). Upregulation of miR-495 contributes to lower methionine adenosyltransferase 1A (MAT1A) expression in HCC through suppressing the translation and increasing the degradation of MAT1A mRNA (32). In murine models of HCC, stable overexpression of miR-495 was demonstrated to promote tumorigenesis and metastasis (32).

miR-495 was demonstrated to have similar functions in gallbladder cancer (GBC) to those it has in breast cancer and HCC. A previous study identified that $\mathrm{PH}$ domain and leucine rich repeat protein phosphatase (PHLPP) is a target of miR-495 (33). PHLPP acts as tumor suppressor and survivin has a strong anti-apoptotic effect in $\operatorname{GBC}(34,35)$. PHLPP can regulate the activity of survivin directly through phosphorylation and nuclear export, or indirectly through 
Table I. Targets, expression level and function of miR-495 in different types of cancer.

\begin{tabular}{llllc}
\hline Type of cancer & Expression of miR-495 & Target gene(s) & Functions of miR-495 & Reference \\
\hline Gallbladder & Up & PHLPP & $\uparrow$ Cell proliferation & $(33)$ \\
Hepatocellular carcinoma & Up & MAT1A & $\uparrow$ Cell proliferation, $\uparrow$ invasion & $(32)$ \\
Glioblastoma multiforme & Down & CDK6 & $\downarrow$ Cell growth & $(31)$ \\
Prostate & Down & N/A & $\downarrow$ Cell growth & $(9)$ \\
Non-small cell lung cancer & Down & MTA3 & $\downarrow$ Drug resistance, $\downarrow$ metastasis & $(30)$ \\
Non-small cell lung cancer & Down & ATP7A & $\downarrow$ Drug resistance & $(44)$ \\
Small cell lung cancer & Down & N/A & $\downarrow$ Drug resistance & $(45)$ \\
Gastric & Down & PRL-3 & $\downarrow$ Invasion, $\downarrow$ metastasis & $(12,29)$ \\
Breast & Up & E-cadherin, REDD1 & $\uparrow$ Cell proliferation, $\uparrow$ invasion & $(13)$ \\
Acute myeloid leukemia & Down & PBX3, MEIS1 & $\downarrow$ Cell proliferation, $\downarrow$ invasion & $(14)$ \\
Chronic myeloid leukemia & Down & MDR1 & $\downarrow$ Drug resistance & $(43)$
\end{tabular}

Up, upregulation; down, downregulation; PHLPP, PH domain and leucine rich repeat protein phosphatase; MAT1A, methionine adenosyltransferase 1A; CDK6, cyclin dependent kinase 6; MTA3, metastasis associated 1 family member 3; ATP7A, ATPase copper transporting $\alpha$; PRL-3, phosphatase of regenerating liver-3; E-cadherin, epithelial-cadherin; REDD1, DNA damage inducible transcript 4; PBX3, pre-B-cell leukemia homeobox 3; MEIS1, Meis homeobox 1; MDR1, ATP binding cassette subfamily B member 1 .

the protein kinase B/AKT serine/threonine kinase (AKT) signaling pathway, leading to the inhibition of apoptosis and promotion of proliferation of GBC cells. It has been proposed that a miR-495/PHLPP/AKT/survivin anti-apoptosis signaling pathway exists (Fig. 2) (33).

Identifying the specific role of miR-495 in tumors remains a challenge. The reason why miR-495 is upregulated in some cancers and downregulated in others remains unclear. Various studies indicate that the expression of miR-495 is associated with V-Ki-ras2 Kirsten rat sarcoma viral oncogene homolog (KRAS) $(36,37)$. miR-495 is upregulated in KRAS-positive adenocarcinomas, such as those of the lung and pancreas $(36,37)$. As miR-495 serves essential roles in numerous cancers, further studies are required to investigate the regulatory mechanisms underlying miRNA-495 expression.

miR-495 in hematological malignancies. Acute myeloid leukemia (AML) is a heterogeneous group of hematopoietic malignancies. Specific gene mutations and chromosomal aberrations are frequently found in AML and contribute greatly to the pathogenesis of the disease. AMLs with MLL rearrangements are associated with intermediate or poor patient survival (38). miRNAs significantly regulate the process of leukemogenesis.

In a large-scale genome-wide study, miRNA expression profiling analysis demonstrated that miR-495 was expressed at a lower level in MLL-rearranged AML compared with non-MLL-rearranged AML and the normal control samples (14). Through targeting pre-b-cell leukemia transcription factor 3 and Meis homeobox 1, miR-495 suppresses MLL-fusion-mediated cell transformation, leukemogenesis and viability, and promotes apoptosis (14). Studies investigating the role of miR-495 in leukemia have suggested that fusion proteins are an essential factor in leukemogenesis. As discussed above, miR-495 was identified to be downregulated by MLL-AF9 in cytological and animal experiments (14). Consequently, in MLL-rearranged AML, fusion proteins are important in modulating the expression of miR-495. Aberrant overexpression or underexpression of miR-495 deregulates target gene expression and signaling pathways to promote leukemogenesis, indicating that miR-495 expression is a prognostic marker for AML.

miR-495 enhances tumor sensitivity to chemotherapy. The occurrence of drug resistance results from multiple processes, such as drug transport and metabolism, DNA synthesis and repair, and cell proliferation and apoptosis $(39,40)$. miRNAs serve a role in increasing chemosensitivity. However, the downregulation of miRNAs whose encoding genes are located on chromosome $14 \mathrm{q} 32.31$ is associated with chemotherapeutic resistance in cancer (41-43).

miR-495 is downregulated in patients and cell lines with drug resistance, indicating that it primarily functions as an anti-tumor factor by increasing chemosensitivity. miR-495, together with other miRNAs encoded by the 14q32.31 gene cluster, may increase chemosensitivity in lung, breast and pancreatic cancer, in addition to leukemia, which could improve survival. In human lung adenocarcinoma cisplatin-resistant A549/CDDP cells, multidrug resistant cell lines of NSCLC, overexpression of miR-495 downregulates ATPase copper transporting $\alpha$ (ATP7A) and enhances sensitivity to cisplatin (CDDP) (44). Knockdown of ATP7A produces similar effects. Additionally, miR-495 could increase intracellular CDDP concentration and overexpression of ATP7A could reduce the effect caused by miR-495 (44).

In small cell lung cancer (SCLC), miR-495, miR-134 and miR-379, whose genes are located in chromosome region $14 q 32.31$, have been demonstrated to increase sensitivity to anti-cancer drugs containing cisplatin, etoposide and doxorubicin (45). It has been proposed that Rho-family GTPase 3 is a potential target of miR-495. Similarly, miR-495 increases chemotherapeutic sensitivity in chronic myeloid leukemia (CML) (43). Numerous 14q32.31 cluster-located miRNAs are downregulated in the stable Adriamycin (ADM) 
-resistant cell line K562/ADM (43). Transfecting K562/ADM cells with miR-495 mimics was demonstrated to increase sensitivity to ADM by downregulating P-glycoprotein expression (43). In pancreatic cancer, garcinol sensitizes cancer cells to gemcitabine by upregulating miR-495 and activating miR-495-associated signaling pathways (37).

As drug resistance is a major problem faced in the treatment of cancer, patients who have a poor response to chemotherapy will benefit from agents that sensitize cancer cells to chemotherapy. The use of miRNA-495 is a promising therapeutic approach for the treatment of multidrug resistant cancers.

The expression profile of miR-495 and its targets, and its roles in cancer are described in Table I. The regulatory roles of miR-495 in disease are primarily determined by its negative activity on the expression of its target mRNAs. At present, the expression and regulation of miRNA-495 has only been studied in certain cancers. Further research is warranted to investigate the role of miRNA-495 in other malignant diseases.

\section{Conclusions}

In the present review, the participation of miR-495 in numerous normal biological processes, including development, immune responses and angiogenesis has been highlighted. In addition, miR-495 serves an essential role in pathological processes, such as inflammation and tumorigenesis. In human cancers, miR-495 is typically downregulated and has an anti-tumorigenic effect. Similarly to other miRNAs encoded by genes located in the 14q32.31 miRNA cluster, miR-495 is able to arrest proliferation, inhibit invasion and induce the apoptosis of cells. Importantly, miR-495 and other miRNAs encoded by this cluster increase the sensitivity of cancer cells to chemotherapy drugs through raising intracellular drug concentration.

The regulation, expression and function of miR-495 remains controversial. miR-495 has been demonstrated to be upregulated and act as an oncogenic factor in breast cancer, hepatocellular carcinoma and gallbladder cancer. At present, the precise mechanisms driving its upregulation or downregulation remain to be elucidated. As discussed in the current review, miR-495 is regulated by the transcription factors EF12 and EF47, in addition to promoter methylation status and the MLL-AF9 oncoprotein. In addition, miR-495 appears to be selectively overexpressed in KRAS-positive adenocarcinomas, where it acts as an oncomiR. Whether KRAS is a determining factor for the upregulation of miR-495 remains unknown. Further studies on miR-495 are required to ascertain the molecular mechanisms underlying the regulation and function of miR-495. Preclinical research will aid in the understanding of the tumor-specific expression pattern of miR-495 and its role in specific types of cancer. In conclusion, present research indicates that miR-495 is a potential diagnostic and prognostic biomarker, and could provide a novel therapeutic approach for the treatment of multidrug resistant cancers.

\section{Acknowledgments}

This present review was supported by the Program for Changjiang Scholars and Innovative Research Teams in Universities (grant no. PCSIRT:1171) and the Science and
Technology Innovative Engineering Project of Shaanxi Province (grant no. 2014KTCL03-01).

\section{References}

1. Bartel DP: MicroRNAs: Genomics, biogenesis, mechanism, and function. Cell 116: 281-297, 2004.

2. Monterisi S, D'Ario G, Dama E, Rotmensz N, Confalonieri S, Tordonato C, Troglio F, Bertalot G, Maisonneuve P, Viale G, et al: Mining cancer gene expression databases for latent information on intronic microRNAs. Mol Oncol 9: 473-487, 2015.

3. Zhang S, Liu Y and Yu B: New insights into pri-miRNA processing and accumulation in plants. Wiley Interdiscip Rev RNA 6: 533-545, 2015.

4. Hammond SM: An overview of microRNAs. Adv Drug Deliv Rev 87: 3-14, 2015.

5. Lewkowicz P, Cwiklińska H, Mycko MP, Cichalewska M, Domowicz M, Lewkowicz N, Jurewicz A and Selmaj KW: Dysregulated RNA-induced silencing complex (RISC) assembly within CNS corresponds with abnormal miRNA expression during autoimmune demyelination. J Neurosci 35: 7521-7537, 2015.

6. Megraw M, Sethupathy P, Corda B and Hatzigeorgiou AG: miRGen: A database for the study of animal microRNA genomic organization and function. Nucleic Acids Res 35 (Database Issue): D149-D155, 2007.

7. Jha P, Agrawal R, Pathak P, Kumar A, Purkait S, Mallik S, Suri V, Chand Sharma M, Gupta D, Suri A, et al: Genome-wide small noncoding RNA profiling of pediatric high-grade gliomas reveals deregulation of several miRNAs, identifies downregulation of snoRNA cluster HBII-52 and delineates H3F3A and TP53 mutant-specific miRNAs and snoRNAs. Int J Cancer 137: 2343-2353, 2015

8. Vinuesa CG, Rigby RJ and Yu D: Logic and extent of miRNA-mediated control of autoimmune gene expression. Int Rev Immunol 28: 112-138, 2009.

9. Formosa A, Markert EK, Lena AM, Italiano D, Finazzi-Agro' E, Levine AJ, Bernardini S, Garabadgiu AV, Melino G and Candi E: MicroRNAs, miR-154, miR-299-5p, miR-376a, miR-376c, miR-377, miR-381, miR-487b, miR-485-3p, miR-495 and miR-654-3p, mapped to the 14q32.31 locus, regulate proliferation, apoptosis, migration and invasion in metastatic prostate cancer cells. Oncogene 33: 5173-5182, 2014.

10. Choi EJ, Kim HB, Baek YH, Kim EH, Pascua PN, Park SJ, Kwon HI, Lim GJ, Kim S, Kim YI and Choi YK: Differential microRNA expression following infection with a mouse-adapted, highly virulent avian H5N2 virus. BMC Microbiol 14: 252, 2014.

11. Guo Z, Wu R, Gong J, Zhu W, Li Y, Wang Z, Li N and Li J: Altered microRNA expression in inflamed and non-inflamed terminal ileal mucosa of adult patients with active Crohn's disease. J Gastroenterol Hepatol 30: 109-116, 2015.

12. Li Z, Zhang G, Li D, Jie Z, Chen H, Xiong J, Liu Y, Cao Y, Jiang M, Le Z and Tan S: Methylation-associated silencing of miR-495 inhibit the migration and invasion of human gastric cancer cells by directly targeting PRL-3. Biochem Biophys Res Commun 456: 344-350, 2015.

13. Hwang-Verslues WW, Chang PH, Wei PC, Yang CY, Huang CK, Kuo WH, Shew JY, Chang KJ, Lee EY and Lee WH: miR-495 is upregulated by E12/E47 in breast cancer stem cells, and promotes oncogenesis and hypoxia resistance via downregulation of E-cadherin and REDD1. Oncogene 30: 2463-2474, 2011.

14. Jiang X, Huang H, Li Z, He C, Li Y, Chen P, Gurbuxani S, Arnovitz S, Hong GM, Price C, et al: MiR-495 is a tumorsuppressor microRNA down-regulated in MLL-rearranged leukemia. Proc Natl Acad Sci USA 109: 19397-19402, 2012.

15. Yang D, Wang G, Zhu S, Liu Q, Wei T, Leng Y, Duan T and Kang J: MiR-495 suppresses mesendoderm differentiation of mouse embryonic stem cells via the direct targeting of Dnmt3a. Stem Cell Res 12: 550-561, 2014.

16. Lee S, Yoon DS, Paik S, Lee KM, Jang Y and Lee JW: microRNA-495 inhibits chondrogenic differentiation in human mesenchymal stem cells by targeting Sox9. Stem Cells Dev 23: 1798-1808, 2014

17. Akiyama H, Chaboissier MC, Martin JF, Schedl A and de Crombrugghe B: The transcription factor Sox9 has essential roles in successive steps of the chondrocyte differentiation pathway and is required for expression of Sox 5 and Sox6. Genes Dev 16: 2813-2828, 2002. 
18. Prévot PP, Augereau C, Simion A, Van den Steen G, Dauguet N, Lemaigre FP and Jacquemin P: Let-7b and miR-495 stimulate differentiation and prevent metaplasia of pancreatic acinar cells by repressing HNF6. Gastroenterology 145: 668-678.e6, 2013.

19. Cattaneo M, Pelosi E, Castelli G, Cerio AM, D'Angiò A, Porretti L, Rebulla P, Pavesi L, Russo G, Giordano A, et al: A miRNA signature in human cord blood stem and progenitor cells as potential biomarker of specific acute myeloid leukemia subtypes. J Cell Physiol 230: 1770-1780, 2015.

20. Welten SM, Bastiaansen AJ, de Jong RC, de Vries MR, Peters EA, Boonstra MC, Sheikh SP, La Monica N, Kandimalla ER, Quax PH and Nossent AY: Inhibition of 14q32 MicroRNAs miR-329, miR-487b, miR-494, and miR-495 increases neovascularization and blood flow recovery after ischemia. Circ Res 115 696-708, 2014

21. Moreira LO, El Kasmi KC, Smith AM, Finkelstein D, Fillon S, Kim YG, Núñez G, Tuomanen E and Murray PJ: The TLR2-MyD88-NOD2-RIPK2 signalling axis regulates a balanced pro-inflammatory and IL-10-mediated anti-inflammatory cytokine response to Gram-positive cell walls. Cell Microbiol 10: 2067-2077, 2008.

22. Chuang AY, Chuang JC, Zhai Z, Wu F and Kwon JH: NOD2 expression is regulated by microRNAs in colonic epithelial HCT116 cells. Inflamm Bowel Dis 20: 126-135, 2014.

23. Cho JH and Brant SR: Recent insights into the genetics of inflammatory bowel disease. Gastroenterology 140: 1704-1712, 2011.

24. Chen C, Li Y, Yu Z, Liu Z, Shi Y, Lewandowska U, Sobczak M Fichna J and Kreis M: Platelet activity in the pathophysiology of inflammatory bowel diseases. Current Drug Targets 16: 219-225, 2015.

25. Layne K, Di Giosa P, Ferro A and Passacquale G: LB03.03: Anti-inflammatory effects of anti-platelet drugs: Implication for atherosclerosis. J Hypertens 33 (Suppl 1): e126, 2015.

26. Hedl M, Lahiri A, Ning K, Cho JH and Abraham C: Pattern recognition receptor signaling in human dendritic cells is enhanced by ICOS ligand and modulated by the Crohn's disease ICOSLG risk allele. Immunity 40: 734-746, 2014.

27. van Engeland M, Derks S, Smits KM, Meijer GA and Herman JG: Colorectal cancer epigenetics: Complex simplicity. J Clin Oncol 29: 1382-1391, 2011.

28. Wang Z, He YL, Cai SR, Zhan WH, Li ZR, Zhu BH, Chen CQ, Ma JP, Chen ZX, Li W and Zhang LJ: Expression and prognostic impact of PRL-3 in lymph node metastasis of gastric cancer: Its molecular mechanism was investigated using artificial microRNA interference. Int J Cancer 123: 1439-1447, 2008.

29. Li Z, Cao Y, Jie Z, Liu Y, Li Y, Li J, Zhu G, Liu Z, Tu Y, Peng G, et al: miR-495 and miR-551a inhibit the migration and invasion of human gastric cancer cells by directly interacting with PRL-3. Cancer Lett 323: 41-47, 2012.

30. Chu H, Chen X, Wang H, Du Y, Wang Y, Zang W, Li P, Li J, Chang J, Zhao G and Zhang G: MiR-495 regulates proliferation and migration in NSCLC by targeting MTA3. Tumour Biol 35 3487-3494, 2014.

31. Chen SM, Chen HC, Chen SJ, Huang CY, Chen PY, Wu TW, Feng LY, Tsai HC, Lui TN, Hsueh C and Wei KC: MicroRNA-495 inhibits proliferation of glioblastoma multiforme cells by downregulating cyclin-dependent kinase 6. World J Surg Oncol 11: 87, 2013.

32. Yang H, Cho ME, Li TW, Peng H, Ko KS, Mato JM and Lu SC: MicroRNAs regulate methionine adenosyltransferase 1A expression in hepatocellular carcinoma. J Clin Invest 123: 285-298, 2013.
33. Liu C, Sun B, An N, Tan W, Cao L, Luo X, Yu Y, Feng F, Li B, Wu M, et al: Inhibitory effect of Survivin promoter-regulated oncolytic adenovirus carrying P53 gene against gallbladder cancer. Mol Oncol 5: 545-554, 2011.

34. Nitsche C, Edderkaoui M, Moore RM, Eibl G, Kasahara N, Treger J, Grippo PJ, Mayerle J, Lerch MM and Gukovskaya AS: The phosphatase PHLPP1 regulates Akt2, promotes pancreatic cancer cell death, and inhibits tumor formation. Gastroenterology 142: 377-387.e1-5, 2012.

35. Qiu Y, Li X, Yi B, Zheng J, Peng Z, Zhang Z, Wu M, Shen F and $\mathrm{Su}$ C: Protein phosphatase PHLPP induces cell apoptosis and exerts anticancer activity by inhibiting Survivin phosphorylation and nuclear export in gallbladder cancer. Oncotarget 6: 19148-19162, 2015

36. Dacic S, Kelly L, Shuai Y and Nikiforova MN: miRNA expression profiling of lung adenocarcinomas: Correlation with mutational status. Mod Pathol 23: 1577-1582, 2010.

37. Parasramka MA, Ali S, Banerjee S, Deryavoush T, Sarkar FH and Gupta S: Garcinol sensitizes human pancreatic adenocarcinoma cells to gemcitabine in association with microRNA signatures. Mol Nutr Food Res 57: 235-248, 2013.

38. Pigneux A, Labopin M, Maertens J, Cordonnier C, Volin L, Socié G, Blaise D, Craddock C, Milpied N, Bacher U, et al: Outcome of allogeneic hematopoietic stem-cell transplantation for adult patients with AML and 11q23/MLL rearrangement (MLL-r AML). Leukemia 29: 2375-2381, 2015.

39. De Mattia E, Cecchin E and Toffoli G: Pharmacogenomics of intrinsic and acquired pharmacoresistance in colorectal cancer: Toward targeted personalized therapy. Drug Resist Updat 20: 39-70, 2015 .

40. Neesse A and Gress TM: Emerging role of microRNAs to tackle drug resistance in pancreatic cancer. Gut 64: 1842-1843, 2015.

41. Laddha SV, Nayak S, Paul D, Reddy R, Sharma C, Jha P, Hariharan M, Agrawal A, Chowdhury S, Sarkar C and Mukhopadhyay A: Genome-wide analysis reveals downregulation of miR-379/miR-656 cluster in human cancers. Biol Direct 8: 10 , 2013.

42. Haller F, von Heydebreck A, Zhang JD, Gunawan B, Langer C, Ramadori G, Wiemann S and Sahin O: Localization- and mutation-dependent microRNA (miRNA) expression signatures in gastrointestinal stromal tumours (GISTs), with a cluster of co-expressed miRNAs located at 14q32.31. J Pathol 220: 71-86, 2010.

43. Xu Y, Ohms SJ, Li Z, Wang Q, Gong G, Hu Y, Mao Z, Shannon MF and Fan JY: Changes in the expression of miR-381 and miR-495 are inversely associated with the expression of the MDR1 gene and development of multi-drug resistance. PLoS One 8: e82062, 2013.

44. Song L, Li Y, Li W, Wu S and Li Z: miR-495 enhances the sensitivity of non-small cell lung cancer cells to platinum by modulation of copper-transporting P-type adenosine triphosphatase A (ATP7A). J Cell Biochem 115: 1234-1242, 2014.

45. Guo L, Liu Y, Bai Y, Sun Y, Xiao F and Guo Y: Gene expression profiling of drug-resistant small cell lung cancer cells by combining microRNA and cDNA expression analysis. Eur J Cancer 46: 1692-1702, 2010. 